Artículo científico

(Original paper)

\title{
DESCRIPCIÓN DE LOS ESTADOS INMADUROS DE PHYLLOPHAGA (PHYTALUS) TOTOREANA Y PHYLLOPHAGA (PHYLLOPHAGA) OPACA DEL NORTE DE SINALOA, MÉXICO
}

\author{
DESCRIPTION OF THE IMMATURE STAGES OF PHYLLOPHAGA (PHYTALUS) \\ TOTOREANA AND PHYLLOPHAGA (PHYLLOPHAGA) OPACA FROM NORTH SINALOA, \\ MEXICO
}

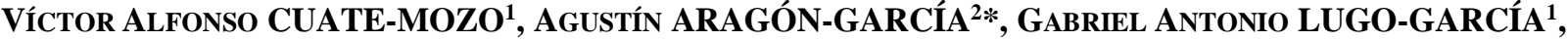 \\ Álvaro ReYes OLIVAS ${ }^{1}$, Miguel ARAGón-SÁNCHEZ², BeTZABeth Cecilia PÉREZ-TORRES ${ }^{2}$
${ }^{1}$ Universidad Autónoma de Sinaloa, Colegio de Ciencias Agropecuarias, Facultad de Agricultura del Valle del Fuerte, Calle 16 y Avenida Japaraqui, C.P. 81110. Juan José Ríos, Ahome, Sinaloa, México. <pneuma@ hotmail.com>; <gabriel_lugo9010@hotmail.com>; <alreo.uas@gmail.com>
${ }^{2}$ Centro de Agroecología, Instituto de Ciencias, Benemérita Universidad Autónoma de Puebla. C.P. 72570. EcoCampus Valsequillo. Edificio VAL 1, Km 1.7 carretera San Baltazar Tetela, San Pedro Zacachimalpa, Puebla, México. <agustin.aragon@correo.buap.mx>; <m.aragon.sanchez@hotmail.com>; <betzabeth.perez@correo.buap.mx>
*Autor corresponsal: <agustin.aragon@ correo.buap.mx> \\ Recibido: 18/10/2019; aceptado: 13/01/2020; publicado en línea: 22/06/2020 \\ Editor responsable: Magdalena Cruz
}

Cuate-Mozo, V. A., Aragón-García, A., Lugo-García, G. A., Reyes Olivas, A., Aragón-Sánchez, M., Pérez-Torres, B. C. (2020) Descripción de los estados inmaduros de Phyllophaga (Phytalus) totoreana y Phyllophaga (Phyllophaga) opaca del norte de Sinaloa, México. Acta Zoológica Mexicana (nueva serie), 36, 1-14.

https://doi.org/10.21829/azm.2020.3612244

RESUMEN. Se presentan las descripciones de larvas de tercer estadio de Phyllophaga (Phytalus) totoreana Morón y Phyllophaga (Phyllophaga) opaca Moser con ejemplares colectados asociados a las raíces de Pennisetum sp. (Poaceae) y al cultivo de maíz (Zea mays L.) (Poaceae) establecidos entre los 118-119 m de altitud en el municipio del Fuerte, Sinaloa, México. Se incluyen ilustraciones de las estructuras características, comentarios sobre las similitudes con larvas de otras especies y una clave taxonómica para la identificación de las larvas de Phyllophaga en México.

Palabras clave: rizófaga; "gallina ciega”; morfología; taxonomía

Cuate-Mozo, V. A., Aragón-García, A., Lugo-García, G. A., Reyes Olivas, A., Aragón-Sánchez, M., Pérez-Torres, B. C. (2020) Description of the immature stages of Phyllophaga (Phytalus) totoreana and Phyllophaga (Phyllophaga) opaca from north Sinaloa, Mexico. Acta Zoológica Mexicana (nueva serie), 36, 1-14.

https://doi.org/10.21829/azm.2020.3612244

ABSTRACT. The descriptions of third stage larvae of Phyllophaga (Phytalus) totoreana Morón and Phyllophaga (Phyllophaga) opaca Moser with collected specimens associated with the roots of Pennisetum 
sp. (Poaceae) and the farming of corn (Zea mays L.) (Poaceae) established between 118-119 $\mathrm{m}$ altitude in the municipality of Fuerte, Sinaloa, Mexico. The pictures of diagnostic characters, comments on similarities with larvae of other species and a taxonomic a key to the larvae of Mexico genera of Phyllophaga is included.

Key words: rhizophagous; "white grubs"; morphology; taxonomy

\section{INTRODUCCIÓN}

La familia Melolonthidae integra las especies del complejo "gallina ciega" en las subtribus Rhizotrogina, Melolonthina y Diplotaxina, siendo la primera la más diversa, con 76 géneros y 1,770 especies que se distribuyen en América, Eurasia, Asia y África (Lacroix, 2014). En 2016, se estimó que el género Phyllophaga registraba 787 especies en las regiones Neártica y Neotropical, repartidas en nueve subgéneros: Phyllophaga sensu stricto, Phytalus, Chirodines, Tostegoptera, Eugastra, Cnemarachis, Clemora, Listrochelus y Chlaenobia, pero con las modificaciones recientes de los análisis cladísticos (RiveraGasperín \& Morón, 2017a; b), se integraron como géneros a Listrochelus y Chlaenobia.

Por esta situación en México, se tiene un registro aproximado de 292 especies dentro del género Phyllophaga, de las cuales se ha generado información sobre la morfología y distribución de los adultos. Solo se conoce la identidad taxonómica del 6\% de los estados inmaduros (19 larvas descritas de las 292 especies del género Phyllophaga) de los cuales, la mayoría reducen el rendimiento de los cultivos agrícolas o forestales por el daño ocasionando al sistema radical (Rivera-Gasperín \& Morón, 2013; 2017a; 2017b; Morón et al., 2016; Lugo et al., 2017). Por ello, es fundamental identificar y separar las especies en estado larvario, para determinar cuáles de ellas generan un impacto económico y, de esta forma, diseñar estrategias de manejo pertinentes con la finalidad de minimizar las pérdidas que ocasionan algunas especies del género Phyllophaga a los cultivos. Sin embargo, debido a la poca información taxonómica, es difícil identificar las especies del género Phyllophaga en estado inmaduro debido a la falta de caracteres morfológicos distintivos. Por lo anterior, el objetivo de este estudio fue describir las larvas de tercer estadio de Phyllophaga (Phyllophaga) opaca (Moser, 1918) y Phyllophaga (Phytalus) totoreana Morón, 2006.

\section{MATERIALES Y MÉTODOS}

Se recolectaron 24 larvas de Ph. opaca en diciembre del 2017 en la Sierra de Balacachi $\left(26^{\circ} 17^{\prime}\right.$ 50.5' N, $108^{\circ} 52^{\prime}$ 46.2' O, $119 \mathrm{msnm}$ ) y 36 larvas de Ph. totoreana en noviembre de 2018 en el canal de conexión dique 6 ( $26^{\circ} 30^{\prime} 36.8^{\prime}$ ' N, $108^{\circ} 37^{\prime}$ 00' O, $118 \mathrm{msnm}$ ), perteneciente al municipio del Fuerte, Sinaloa, México, en bosque tropical caducifolio. Las larvas fueron colectadas manualmente de muestras de suelos de $30 \times 30 \times 30 \mathrm{~cm}$ (Lavelle et al., 1981) y se depositaron en recipientes de plástico de un litro de capacidad con suelo del mismo sitio como sustrato. Se transportaron al laboratorio de la Colección Entomológica del Valle del Fuerte, Universidad Autónoma de Sinaloa (CEVF-UAS). Un 30\% de las larvas se fijaron en solución Pampel y cuatro días después se colocaron en solución acuosa de etanol al $70 \%$ para su conservación. El 70\% de las larvas colectadas se mantuvieron vivas para obtener la relación larva-adulto y de este modo verificar su identidad. Las larvas que se conservaron vivas se mantuvieron en recipientes de polietileno con suelo del sitio de colecta, que se esterilizó previamente (Aragón \& Morón, 2004). Los recipientes se colocaron en una cámara de cría a temperatura de $26 \pm 2{ }^{\circ} \mathrm{C}$ y humedad relativa de $70 \pm 10 \%$.

Las observaciones e ilustraciones se hicieron con un estereomicroscopio Carl Zeiss ${ }^{\circledR}$ y una cámara lúcida. Los caracteres y la terminología utilizada en las descripciones fueron tomadas de lo propuesto por Böving (1936). Los ejemplares estudiados se depositaron en la Colección Entomológica de la Facultad de Agricultura del Valle del Fuerte. Para la identificación de las larvas de tercer estadio de Phyllophaga en 
México, se modifica la clave taxonómica realizada por Morón et al. (2016), en la cual se eliminan las especies que pertenecen a los géneros de Triodonyx, Listrochelus y Chlaenobia; agregando caracteres que presentan las especies de Ph. totoreana y Ph. opaca.

\section{RESULTADOS Y DISCUSIÓN}

\section{Descripción de la larva de tercer estadio Phyllophaga (Phytalus) totoreana Morón, 2006} (Figs. 1-12)

Cabeza (Fig. 1). Anchura máxima de la cápsula cefálica, 3.70-3.83 mm. Superficie del cranium lisa, parda amarillenta. Frente con $1+1$ sedas en la región frontal exterior, $1+1$ sedas en la región frontal posterior; con nueve sedas marginales anteriores; con $2+2$ sedas en el ángulo anterior de la frente; el resto de la superficie craneal con 4-5 sedas dorsoepicraneales y ocho paraocelares a cada lado. Sin ocelos. Clípeo con dos sedas centrales y dos laterales. Labro asimétrico, con los márgenes laterales redondeados, con nueve sedas posteriores, dos centrales, $7+7$ laterales y cuatro anteriores. Epifaringe (Fig. 2) con 1.34-1.36 mm de anchura y 1.29-1.31 mm de longitud, con zygum y epizygum largo e irregular, pardo obscuro; haptomerum prominente con ocho heli dispuestos en dos hileras trasversales; cada plegmatium formado con 11-13 plegmata anchos; cada acanthoparia con 12-13 sedas espiniformes recurvadas; proplegmatium marcado por 7-10 plegmatas espaciados; dexiophoba presente, irregular, que se extiende hacia el cono sensorial; laeophoba larga y extendida desde el extremo interno de la laeotorma hacia la chaetoparia izquierda; dexiotorma casi recta y larga $a_{2}$ con el extremo mesial adelgazado; laeotorma corta y ancha; epitorma presente; apotorma indefinido; pternotorma ancha y larga; haptolachus con un cono sensorial agudo desplazado hacia la placa esclerosada y parcialmente cubierto por la dexiophoba; crepis estrecho y diferenciado; chaetoparia izquierda con 57-61 sedas; chaetoparia derecha con 71-75 sedas. Mandíbula derecha con una lámina cortante distal, separada por un diente incisivo proximal y por una escotadura incisiva. Márgenes internos sin dientes. Área molar con dos lóbulos desarrollados $\left(\mathrm{M}_{1-2}\right)$, el lóbulo distal $\mathrm{M}_{1}$ poco desarrollado; sin área estriduladora en vista ventral (Fig. 3a); proceso ventral bien desarrollado, con 10-12 sedas en la región basolateral; en vista dorsal (Fig. 3b) el calx se encuentra prominente y la brustia moderadamente setosa. Mandíbula izquierda con un área sensorial y tres dientes $\left(S_{1}, S_{2}\right.$ y $\left.S_{3}\right), S_{3}$ separado de $S_{2}$ por una escotadura incisiva; lóbulo distal $\left(\mathrm{M}_{1}\right)$ bien desarrollado; sin área estriduladora en vista ventral (Fig. 4a), proceso ventral prominente, región basolateral con 7-8 sedas; en vista dorsal (Fig. 4b) el acia es prominente, brustia moderadamente setosa. Maxilas (Figs. 5,6) con un uncus terminal en la galea y cuatro unci alineados en la lacinia. Palpos maxilares con cuatro palpómeros, el último palpómero el doble de largo que el segundo. Área estriduladora maxilar con 12 dientecillos. Escleroma hipofaríngeo (Fig. 7) asimétrico, con el lado derecho reducido y el izquierdo prominente, el lóbulo izquierdo con ocho sedas, con una fila de sedas delgadas; el lóbulo derecho sin sedas, glosa con 25-27 sedas espiniformes. Último artejo antenal con un área sensorial ovalada en vista dorsal (Fig. 8) y dos áreas sensoriales en vista ventral (Fig. 8a).

Tórax. Los estigmas respiratorios (Fig. 9) miden $0.26-0.28 \mathrm{~mm}$ de largo por 0.16-0.18 $\mathrm{mm}$ de ancho; placa respiratoria amarillo rojiza, uniformemente recurvados en forma de " $\mathrm{C}$ ", la distancia entre los lóbulos de la placa respiratoria es menor que el diámetro dorso ventral de la bulla redondeada. Pronoto amarillo cremoso, con escleroma lateral semiovalado a cada lado, con 14 sedas en la periferia y cuatro sedas centrales. Proprescudo con 26 sedas largas y medianas de forma irregular; proescudo con 19 sedas largas y medianas situadas irregularmente; mesoprescudo con 14 sedas largas y medianas dispuesta irregularmente; mesoescudo con 43 sedas largas y medianas; mesoescutelo con siete sedas largas; metaprescudo con nueve sedas largas de forma irregular; metaescudo con 48 sedas largas y medianas; metaescutelo con nueve sedas largas de forma irregular. Uñas tarsales pro y mesotoráxicas (Fig. 10a, b) similares, alargadas y agudas con dos sedas prebasales; uñas metatorácicas reducidas (Fig. 10c). 


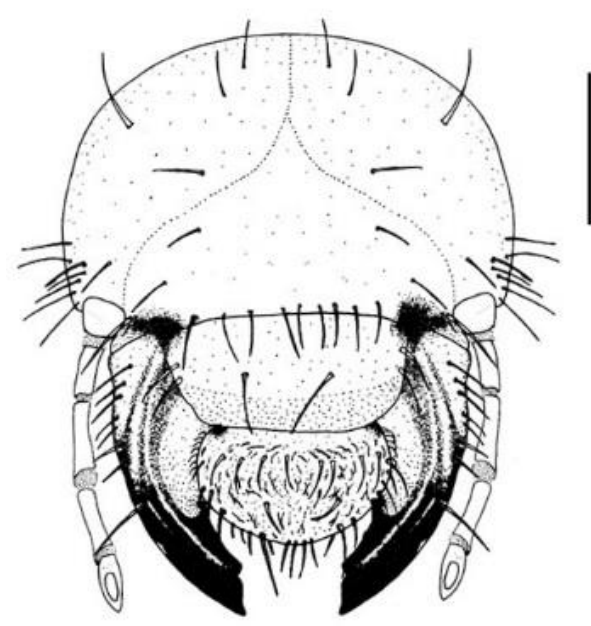

1

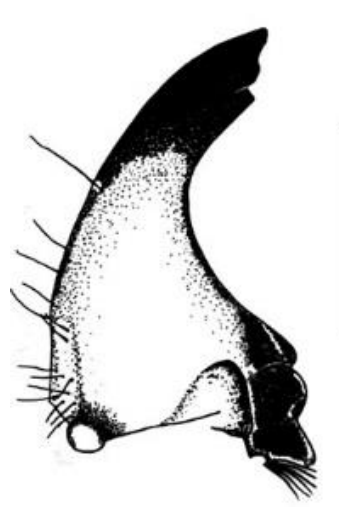

$3 a$

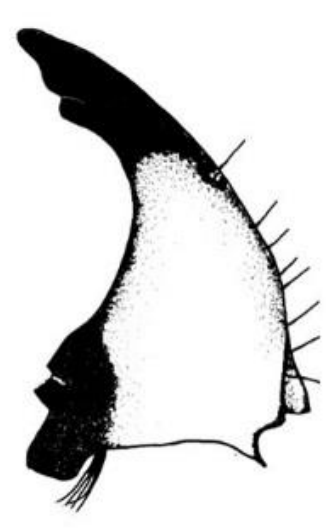

$3 b$

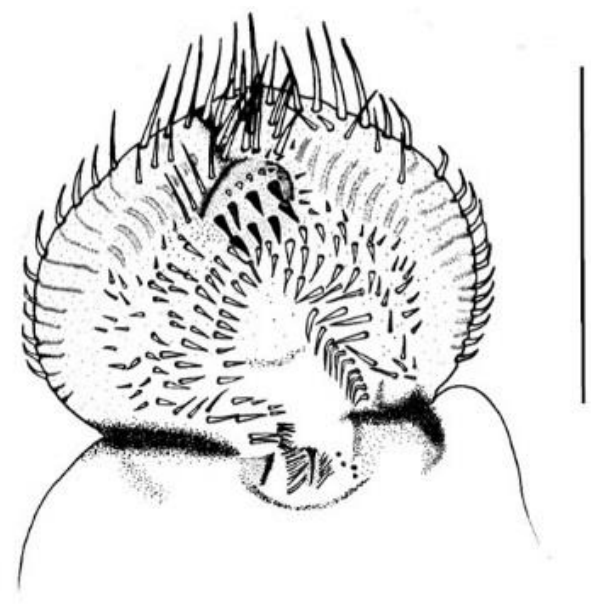

2

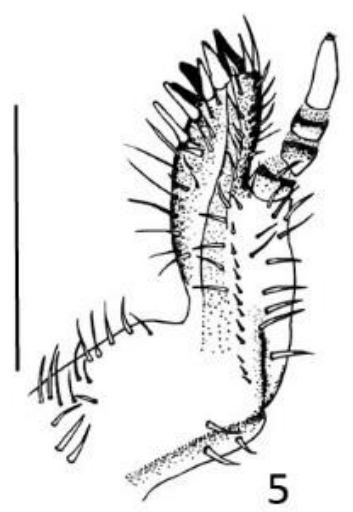

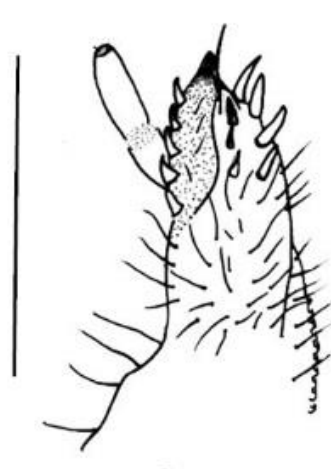

6
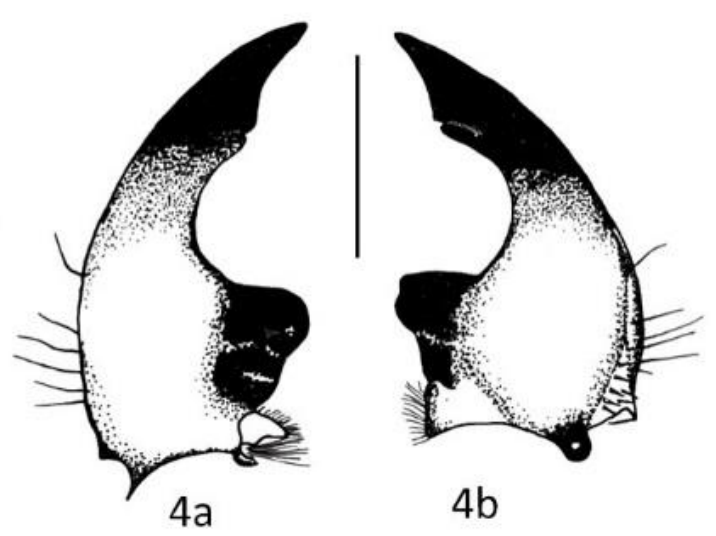

$4 b$

Figuras 1-7. Larva de tercer estadio de Phyllophaga (Phytalus) totoreana. 1) Vista frontal de la cabeza. 2) Aspecto ventral de la epifaringe. Mandíbula derecha: 3a) Vista ventral. 3b) Vista dorsal. Mandíbula izquierda: 4a) Vista ventral. 4b) Vista dorsal. 5) Maxila derecha, dorsal. 6) Maxila vista mesial del ápice de la maxila derecha. 7) hipofaringe. Líneas de escala $=1 \mathrm{~mm}$. 


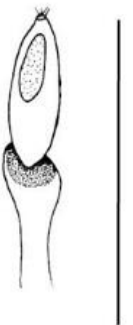

$8 a$

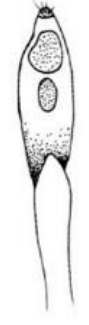

$8 \mathrm{~b}$

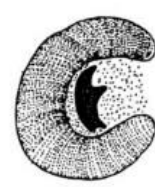

9

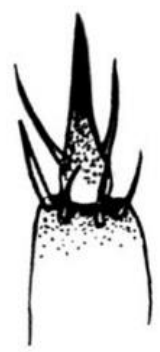

$10 \mathrm{a}$
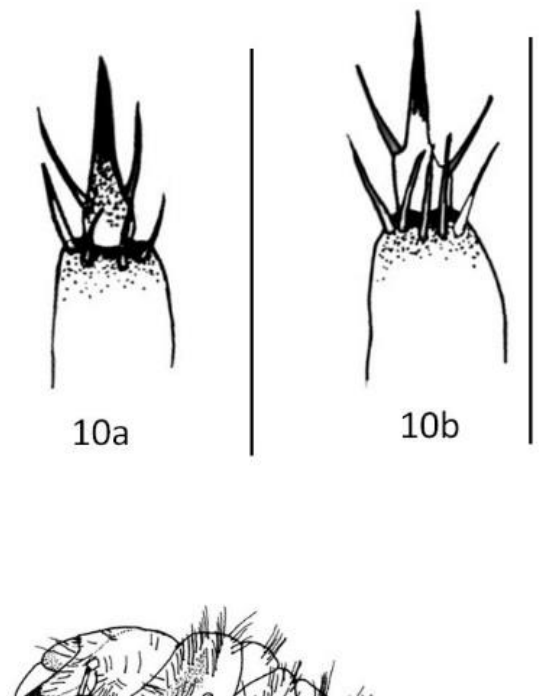
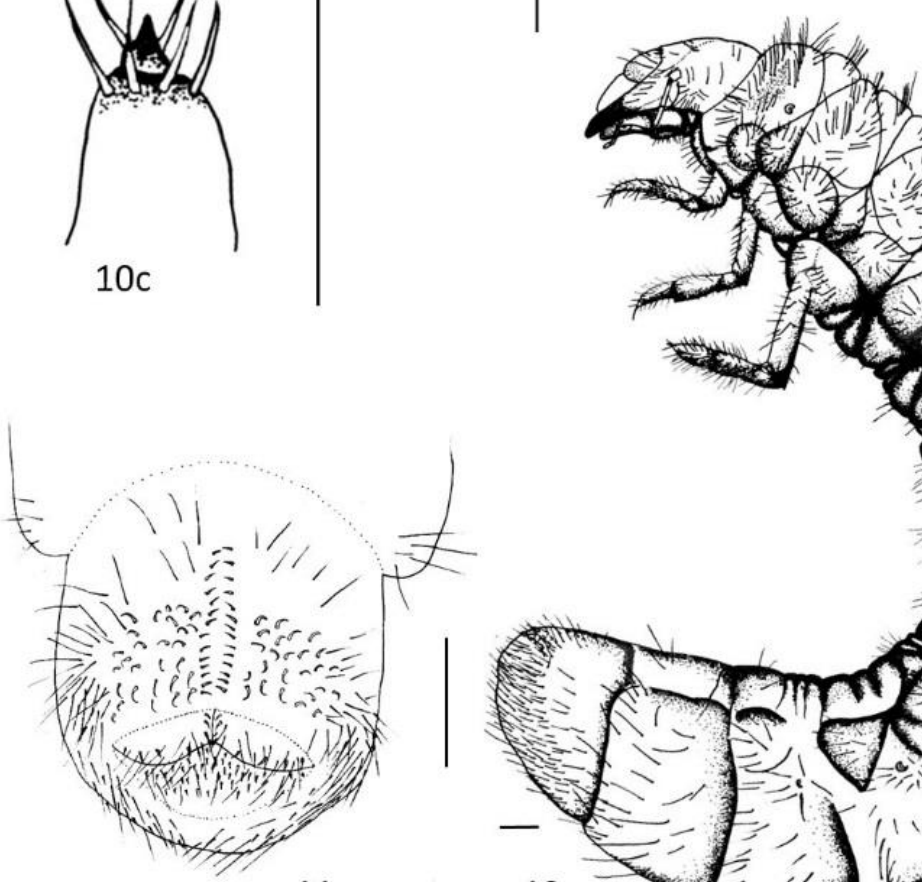

11

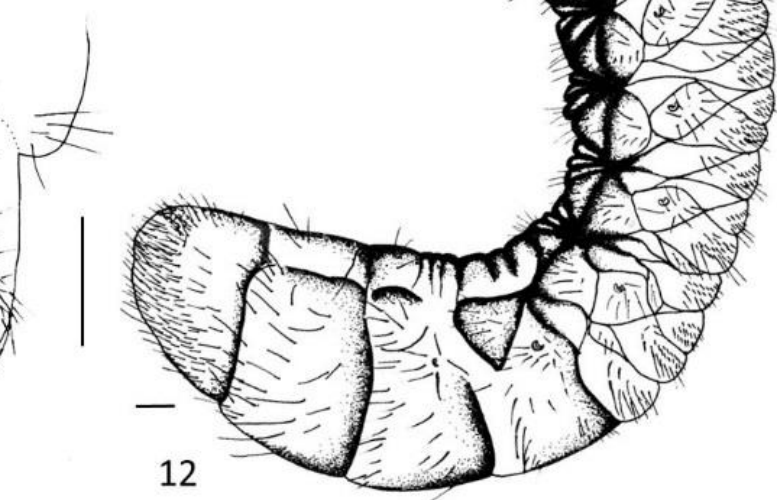

Figuras 8-12. Larva de tercer estadio de Phyllophaga (Phytalus) totoreana. Antenas: 8a) Vista dorsal. 8b) Vista ventral. 9) Estigma respiratorio protorácico. Uñas: 10a) Protarácicas. 10b) Mesotorácicas. 10c) Metatorácicas. 11) Raster. 12) Larva. Líneas de escala $=1 \mathrm{~mm}$, excepto en Fig. $9(=0.5 \mathrm{~mm})$.

Abdomen. Placa respiratoria amarillo rojiza, recurvada en forma de "C". Estigma de los segmentos I-VII con $0.26 \mathrm{~mm}$ de largo por $0.16 \mathrm{~mm}$ de ancho; estigma del segmento VIII reducido en tamaño con respecto a los estigmas mencionados previamente, con diámetro de $0.13 \mathrm{~mm}$ de largo por $0.12 \mathrm{~mm}$ de ancho. Segmento I: proescudo con 13 sedas medianas y largas; escudo con 27 sedas largas esparcidas en hileras transversales irregulares; subescutelo con cinco sedas largas y medianas; escutelo con seis sedas largas y medianas, y 13 sedas espiniformes esparcidas en hileras transversales irregulares. Segmento II: proescudo con tres sedas largas y medianas, 19 sedas espiniformes esparcidas en hileras transversales irregulares; 
escudo con 12 sedas medianas y largas, 38 sedas espiniformes esparcidas en hileras transversales irregulares; subescutelo con seis sedas medianas y largas; escutelo con cuatro sedas medianas y largas, 25 sedas espiniformes esparcidas en hileras transversales irregulares. Segmento III: proescudo con cuatro sedas medianas y largas, 25 sedas espiniformes esparcidas en hileras transversales irregulares; escudo con ocho sedas medianas y largas, 44 sedas espiniformes esparcidas en hileras transversales irregulares; subescutelo con seis sedas largas; escutelo con cuatro sedas medianas y largas, 29 sedas espiniformes esparcidas en hileras transversales irregulares. Segmento IV: proescudo con tres sedas medianas y largas, 23 sedas espiniformes esparcidas en hileras transversales irregulares; escudo con 12 sedas medianas y largas, 36 sedas espiniformes esparcidas en hileras transversales irregulares; subescutelo con cuatro sedas largas; escutelo con seis sedas medianas y largas, 32 sedas espiniformes esparcidas en hileras transversales irregulares. Segmento V: proescudo con cuatro sedas medianas y largas, 29 sedas espiniformes esparcidas en hileras transversales irregulares; escudo con 10 sedas medianas y largas, 39 sedas espiniformes esparcidas en hileras transversales irregulares; subescutelo con cuatro sedas largas; escutelo con cuatro sedas medianas y largas, 27 sedas espiniformes esparcidas en hileras transversales irregulares. Segmento VI: proescudo con cuatro sedas medianas y largas, 27 sedas espiniformes esparcidas en hileras transversales irregulares; escudo con 11 sedas medianas y largas, 29 sedas espiniformes esparcidas en hileras transversales irregulares; subescutelo con cuatro sedas largas; escutelo con seis sedas largas y 13 sedas espiniformes esparcidas en hileras transversales irregulares. Segmento VII: proescudo con siete sedas medianas y largas, 12 sedas espiniformes esparcidas en hileras transversales irregulares; el resto del segmento con 16 sedas largas y medianas. Segmento VIII: 20 sedas medianas y largas esparcidas de forma irregulares, sin sedas espiniformes. Segmento IX: combinación de 25 sedas medianas y largas esparcidas en forma irregulares. Segmento X: abundantes sedas medianas y largas, algunas sedas cortas dispersas, más abundantes hacia los lados. Venter de los segmentos I-VII con 13 sedas largas distribuidas transversalmente; segmento VIII con cinco sedas largas; segmento IX: con dos sedas largas. Raster (Fig. 11) con septula y un par de palidia longitudinales que se aproximan al borde anal inferior, paralelos entre sí, alcanza una longitud de $1.33-1.38 \mathrm{~mm}$ y se encuentran separadas $0.13-0.14 \mathrm{~mm}$ en su parte central; cada palidium está formado por 12-14 pali. Tegilla con 23-24 sedas espiniformes. Campus con 10 sedas largas y finas. Barbula moderadamente setosa, con 13 sedas. Labio anal dorsal con 42 sedas espiniformes y 16 sedad largas hamate. Labio anal ventral con dos sedas gruesas espiniformes, 22 sedas largas y 42 sedas cortas espiniformes. Abertura anal en forma de "Y". Longitud total dorsal aproximada del cuerpo, 27-34 mm (Fig. 12).

Comentarios taxonómicos: La larva de $P$ h. (Ph.) totoreana, se distingue de la larva de Phyllophaga ravida Blanchard y Phyllophaga polyphylla Bates por la presencia de zygum en la epifaringe y porque la cápsula cefálica se encuentra reducida con nueve sedas anterofrontales, cada palidium formado por 12-14 pali espiniformes cortos y afilados; mientras que $P h$. ravida tiene 7-12 sedas anterofrontales y su palidium está formado por 10-14 pali, Ph. polyphylla presenta 8-9 sedas frontales anteriores y cada palidium con 13-16 pali.

Observaciones de sus hábitos y distribución: Los adultos son poco comunes y se han colectado al Noroeste de México, en el estado de Sinaloa cerca de bosques tropicales caducifolios y matorrales establecidos a $25 \mathrm{~m}$ de altitud. Lugo-García et al. (2013) registran la especie en la presa Josefa Ortiz de Domínguez (5 ejemplares) en agosto $\left(26^{\circ} 25^{\prime} \mathrm{N}, 108^{\circ} 43^{\prime} \mathrm{O}\right)$ perteneciente al municipio del Fuerte, asociados a bosque espinoso; en el Cerro de las Microondas (2) en agosto ( $\left.26^{\circ} 19^{\prime} \mathrm{N}, 108^{\circ} 53^{\prime} \mathrm{O}\right)$; en la Bolsa de Tosalibampo (1) en julio ( $\left.25^{\circ} 59^{\prime} \mathrm{N}, 109^{\circ} 06^{\prime} \mathrm{O}\right)$ pertenecientes al municipio de Ahome con vegetación

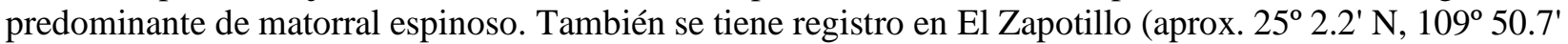
O) perteneciente al Municipio de Mocorito (3) julio, (1) agosto (Morón, 2006). Son atraídas por la luz de vapor de mercurio blanca y luz negra.

Material examinado: Se revisaron 11 larvas de tercer estadio, colectadas en México: Sinaloa, El Fuerte, Canal de conexión dique 6, por V.A. Cuate M. y G. A. Lugo-García, en la siguiente fecha: 10-XI-2018. Asociadas a las raíces de Pennisetum sp. 


\section{Descripción de la larva de tercer estadio Phyllophaga (Phyllophaga) opaca Moser} (Figs. 13-24)

Cabeza (Fig. 13). Anchura máxima de la cápsula cefálica, 5.7-5.9 mm. Superficie del cranium lisa, parda amarillenta. Frente con 1+1 sedas en la región frontal exterior, $2+2$ sedas en la región frontal posterior; con 17 sedas en la región frontal anterior; con 1+1 sedas en el ángulo anterior de la frente; el resto de la superficie craneal con $8+8$ sedas dorso-epicraneales y 13 sedas paraocelares a cada lado, sin ocelos. Clípeo con dos sedas centrales; seis sedas laterales a cada lado. Labro asimétrico, con los márgenes laterales redondeados con 13 sedas posteriores, seis sedas centrales; $6+6$ sedas laterales y dos sedas frontales. Epifaringe (Fig. 14) con 2.5-2.6 mm de anchura y 2.01-2.02 $\mathrm{mm}$ de longitud, con zygum y epizygum largo e irregular, pardo oscuro; haptomerum prominente, con 15 heli dispuestos en tres hileras trasversales; cada plegmatium formado 10-12 plegmata anchos; cada acanthoparia con 11-14 sedas espiniformes recurvadas; proplegmatium marcado por 12-14 plegmatas espaciados; dexiophoba presente, irregular, continua extendiéndose hacia el cono sensorial; laeophoba larga y extendida desde el extremo interno de la laeotorma hacia la chaetoparia izquierda; dexiotorma casi recta y larga, ancha en la parte media y con el extremo mesial adelgazado; laeotorma corta y ancha; epitorma presente, apotorma indefinido; pternotorma ancha y larga; haptolachus con un cono sensorial agudo desplazado hacia la placa esclerosada y parcialmente cubierto por la dexiophoba; crepis estrecho y diferenciado; chaetoparia izquierda con 71-77 sedas; chaetoparia derecha con 98-105 sedas. Mandíbula derecha con una lámina cortante distal, separada por un diente incisivo proximal y por una escotadura incisiva. Márgenes internos sin dientes. Área molar con tres lóbulos desarrollados ( $\left.\mathrm{M}_{1-2-3}\right)$, el lóbulo distal $\mathrm{M}_{1}$ poco desarrollado; sin área estriduladora en vista ventral (Fig. 15a), proceso ventral bien desarrollado, con 9-10 sedas en la región basolateral; en vista dorsal (Fig. 15b), el calx se encuentra prominente y la brustia moderadamente setosa. Mandíbula izquierda con un área sensorial y tres dientes $\left(S_{1}, S_{2}\right.$ y $\left.S_{3}\right), S_{3}$ separado de $S_{2}$ por una escotadura incisiva, lóbulo distal $\left(M_{1}\right)$ bien desarrollado; sin área estriduladora en vista ventral (Fig. 16a), proceso ventral prominente, región basolateral con 8-9 sedas; en vista dorsal (Fig. 16b), el acia es promiente y la brustia moderadamente setosa. Maxilas (Figs. 17, 18) con un uncus terminal en la galea y cuatro unci alineados en la lacinia. Palpos maxilares con cuatro palpómeros, el último palpómero el doble de largo que el tercero. Área estriduladora maxilar con 12 dientecillos. Escleroma hipofaríngeo (Fig. 19) asimétrico, con el lado derecho reducido y el izquierdo prominente, el lóbulo izquierdo con nueve sedas, con una fila de sedas delgadas; lóbulo derecho con tres sedas, glosa con 43-46 sedas espiniformes. Último artejo antenal con una área sensorial ovalada en vista dorsal (Fig. 20a) y dos áreas sensoriales en vista ventral (Fig. 20b).

Tórax. Los estigmas respiratorios (Fig. 21) miden $0.35-0.37 \mathrm{~mm}$ de largo por $0.32-0.34 \mathrm{~mm}$ de ancho; placa respiratoria amarillo rojiza, uniformemente recurvados en forma de " $\mathrm{C}$ ", la distancia entre los lóbulos de la placa respiratoria es menor que el diámetro dorso ventral de la bulla redondeada. Pronoto con escleroma lateral semiovalado a cada lado, amarillo cremoso, con 24 sedas en la periferia. Proprescudo con 30 sedas largas y medianas de forma irregular; proescudo con 10 sedas largas y medianas irregularmente situadas; mesoprescudo con nueve sedas largas y medianas dispuesta irregularmente; mesoescudo con 43 sedas largas y medianas; mesoescutelo con 11 sedas largas; metaprescudo con 10 sedas largas de forma irregular; metaescudo con 42 sedas largas y medianas; metaescutelo con 10 sedas largas de forma irregular. Uñas tarsales pro y mesotoracicas (Fig. 22a, b) similares, alargadas, agudas, con dos sedas prebasales; uñas metatoracicas reducidas (Fig. 22c). 


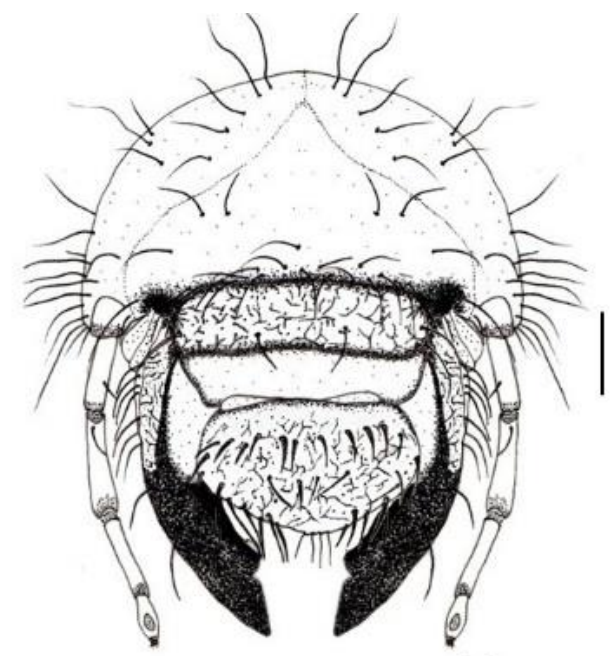

13

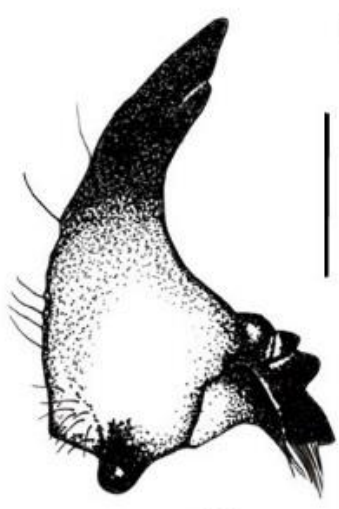

$15 a$

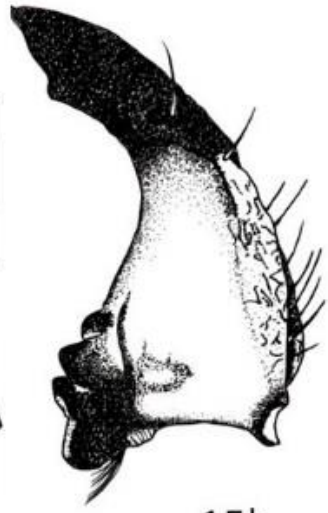

$15 b$

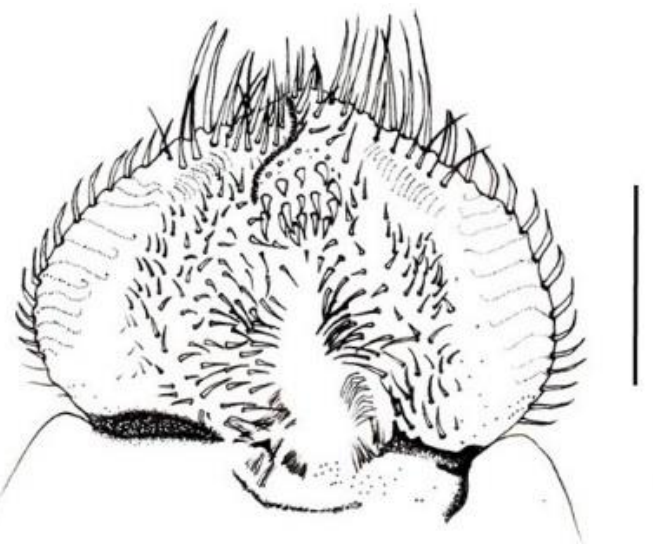

14

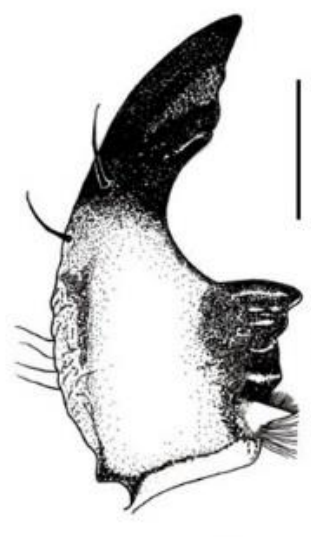

$16 a$

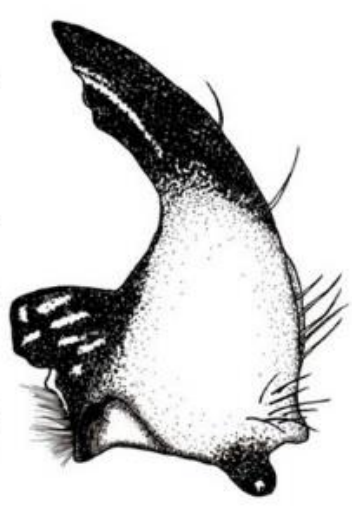

$16 b$

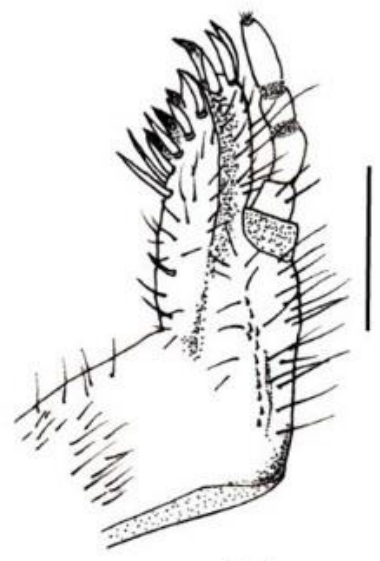

17

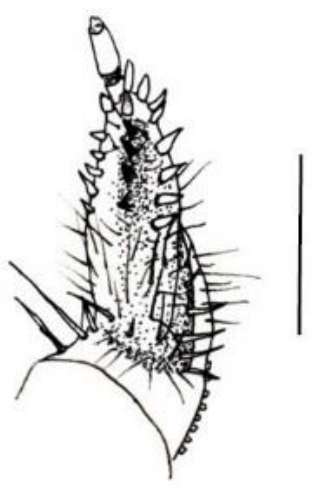

18

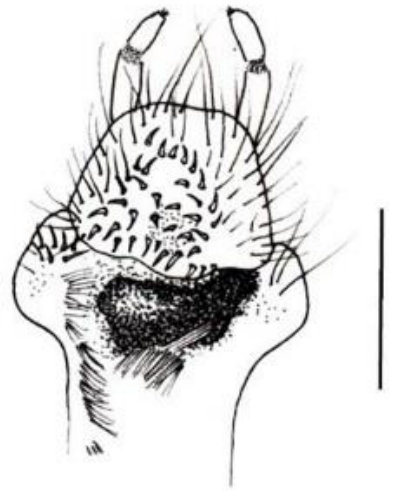

19

Figuras 13-19. Larva de tercer estadio de Phyllophaga (Ph.) opaca. 13) Vista frontal de la cabeza. 14) Aspecto ventral de la epifaringe. Mandíbula derecha: 15a) Vista ventral. 15b) Vista dorsal. Mandíbula izquierda: 16a) Vista ventral. 16b) Vista dorsal. 17) Maxila derecha, dorsal. 18) Maxila vista mesial del ápice de la maxila derecha. 19) hipofaringe. Líneas de escala $=1 \mathrm{~mm}$. 


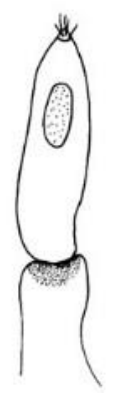

$20 a$

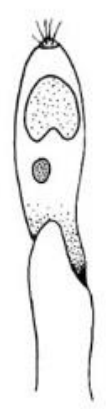

$20 b$

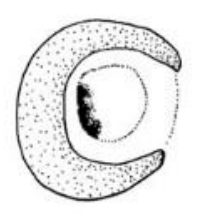

21

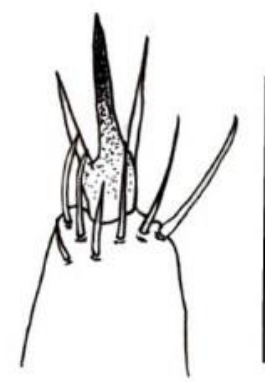

$22 a$

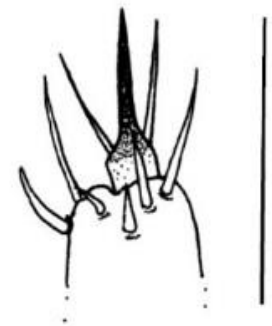

$22 b$

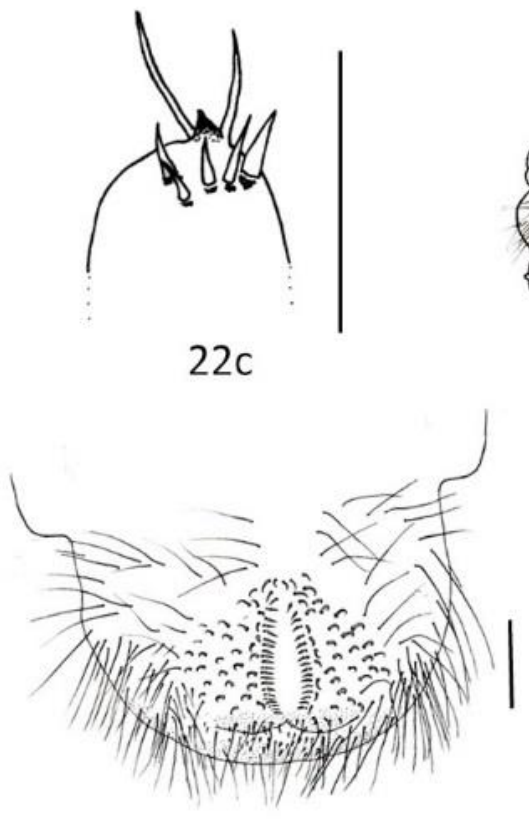

23

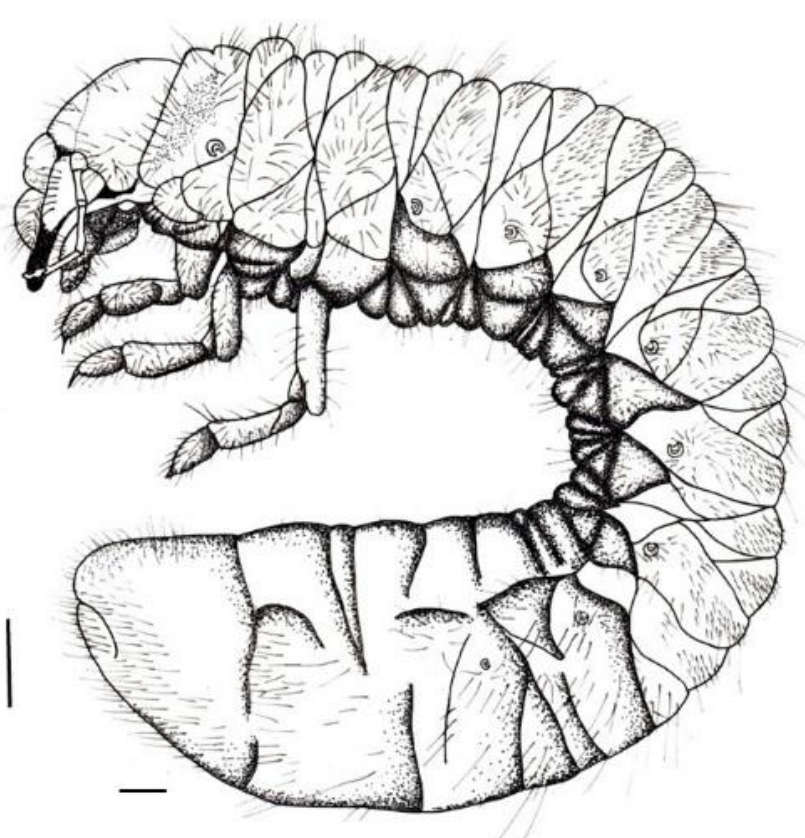

24

Figuras 20-24. Larva de tercer estadio de Phyllophaga (Ph.) opaca. Antenas: 20a) Vista dorsal. 20b) Vista ventral. 21) Estigma respiratorio protorácico. Uñas: 22a) Protarácicas. 22b) Mesotorácicas. 22c) Metatorácicas. 23) Raster. 24) Larva. Líneas de escala $=1 \mathrm{~mm}$, excepto en Fig. 21 (=0.5 mm).

Abdomen. Placa respiratoria amarillo rojiza, regularmente recurvada en forma de "C". Estigma del segmento I con $0.34 \mathrm{~mm}$ de largo por $0.28 \mathrm{~mm}$ de ancho; estigmas de los segmentos II-VII con $0.31 \mathrm{~mm}$ de largo por $0.28 \mathrm{~mm}$ de ancho; estigma del segmento VIII reducido en tamaño con respecto a los estigmas mencionados previamente, con $0.22 \mathrm{~mm}$ de largo por $0.18 \mathrm{~mm}$ de ancho. Segmento I: proescudo con 14 sedas medianas y largas; escudo con 36 sedas largas esparcidas en hileras transversales irregulares y 15 sedas espiniformes; subescutelo con tres sedas largas y medianas; escutelo con cinco sedas largas y medianas, con 30 sedas espiniformes esparcidas en hileras transversales irregulares. Segmento II: proescudo con cuatro sedas largas y medianas, 20 sedas espiniformes esparcidas en hileras transversales irregulares; escudo con 25 sedas medianas y largas, 76 sedas espiniformes esparcidas en hileras transversales irregulares; subescutelo con 13 sedas medianas y largas; escutelo con ocho sedas medianas y largas, 48 sedas espiniformes esparcidas en hileras transversales irregulares. Segmento III: proescudo con dos sedas 
medianas y largas, 30 sedas espiniformes esparcidas en hileras transversales irregulares; escudo con 12 sedas medianas y largas, 82 sedas espiniformes esparcidas en hileras transversales irregulares; subescutelo con 16 sedas largas; escutelo con seis sedas medianas y largas, 52 sedas espiniformes esparcidas en hileras transversales irregulares. Segmento IV: proescudo con dos sedas medianas y largas, 30 sedas espiniformes esparcidas en hileras transversales irregulares; escudo con 12 sedas medianas y largas, 86 sedas espiniformes esparcidas en hileras transversales irregulares; subescutelo con 16 sedas largas; escutelo con seis sedas medianas y largas, 54 sedas espiniformes esparcidas en hileras transversales irregulares. Segmento V: proescudo con dos sedas medianas y largas, 36 sedas espiniformes esparcidas en hileras transversales irregulares; escudo con 12 sedas medianas y largas, 76 sedas espiniformes esparcidas en hileras transversales irregulares; subescutelo con 10 sedas largas; escutelo con ocho sedas medianas y largas, 54 sedas espiniformes esparcidas en hileras transversales irregulares. Segmento VI: proescudo con dos sedas medianas y largas, 46 sedas espiniformes esparcidas en hileras transversales irregulares; escudo con 12 sedas medianas y largas, 64 sedas espiniformes esparcidas en hileras transversales irregulares; subescutelo con ocho sedas largas; escutelo con 12 sedas largas y 42 sedas espiniformes esparcidas en hileras transversales irregulares. Segmento VII: proescudo con 16 sedas medianas y largas, seis sedas espiniformes esparcidas en hileras transversales irregulares; el resto del segmento con 24 sedas largas y medianas. Segmento VIII: con 21 sedas medianas y largas esparcidas en forma irregulares, sin sedas espiniformes. Segmento IX: con una combinación de 25 sedas medianas y largas esparcidas en forma irregulares. Segmento X: con abundantes sedas medianas y largas, algunas sedas cortas dispersas, más abundantes hacia los lados. Venter de los segmentos I-III con 12 sedas largas distribuidas transversalmente. Segmentos VI-V con 10 sedas largas; segmentos del VI-VIII con cuatro sedas; segmento IX con dos sedas largas. Raster (Fig. 23) con septula y un par de palidia longitudinales que se aproximan al borde anal inferior, poco convergentes en la parte anterior, alcanza una longitud de $1.43-1.45 \mathrm{~mm}$ y se encuentran separadas de $0.26-0.28 \mathrm{~mm}$ en su parte central, cada palidium está formado por 17-20 pali. Tegilla con 30 a 34 sedas espiniformes. Campus con 17 sedas largas y finas. Barbula moderadamente setosa con 13 sedas. Labio anal dorsal con 52 sedas espiniformes y 28 sedas largas hamate. Labio anal ventral con cuatro sedas gruesas espiniformes, 10 sedas largas y 13 sedas cortas espiniformes. Abertura anal en forma de "Y". Longitud total dorsal aproximada del cuerpo 3.1-3.6 mm (Fig. 24).

Comentarios taxonómicos: La larva es parecida a la de Phyllophaga multipora y se distingue sobre todo por la presencia de 6-8 sedas anterofrontales, cada palidium está formado por 18-23 pali espiniformes (Ramírez-Salinas \& Castro-Ramírez, 2015); mientras que Ph. opaca tiene 19 sedas anterofrontales y su palidium está formado por 17-20 pali.

Observaciones de sus hábitos y distribución: Phyllophaga (Ph.) opaca (Moser) es una especie rizófaga, de la cual no se han reportado daños al sistema radical de cultivos de la región. Está registrada para los estados de Chihuahua, Michoacán, Nayarit, Sinaloa y Sonora (Morón, 2003). Los adultos se han observado consumiendo el follaje de Pithecellobium dulce (Fabaceae), Helianthus annuus (Asteraceae), Funastrum cynanchoides (Asclepiadaceae), Parkisonia aculeata (Fabaceae), Melochia piramidata (Esterculiaceae), Delonix regia (Fabaceae), Caesalpinia palmeri (Fabaceae), Ficus thonningii (Moraceae), Acacia farnesiana (Fabaceae), Amaranthus spinosus (Amarantaceae), Parthenium argentatum (Asteraceae), Ehretia tinifolia (Boraginaceae), Psidium guajava (Myrtaceae), Baccharis salicifolia (Asteraceae) y Fouquieria macdougalli (Fouqueriaceae), y se han capturado en mayor abundancia en el estado de Sinaloa en el Rancho Olguín ( $\left.26^{\circ} 25^{\prime} \mathrm{N}, 108^{\circ} 35^{\prime} \mathrm{O}\right)$ (1,198 ejemplares) en agosto, Los Terreros (26 $\left.21^{\prime} \mathrm{N}, 108^{\circ} 42^{\prime} \mathrm{O}\right)$ (129) en agosto, en Jahuara II ( $\left.26^{\circ} 13^{\prime} \mathrm{N}, 108^{\circ} 57^{\prime} \mathrm{O}\right)$ (243) en julio y agosto, pertenecientes al municipio del Fuerte, así como en Santa Ana (26 $16^{\circ} \mathrm{N}, 108^{\circ} 19^{\prime}$ O) (17) y Agua Caliente Grande (26 $22^{\circ}$ N, $108^{\circ} 29^{\prime}$ O) (1), correspondientes al municipio de Choix (Lugo-García et al., 2011, 2013, 2017), con frecuencia son atraídos con luz fluorescente negra y blanca. 
Material examinado: Se revisaron siete larvas de tercer estadio, colectadas en México: Sinaloa, El Fuerte, Sierra de Balacachi, por G. A. Lugo-García y V. A. Cuate M., en la siguiente fecha: 04-XII-2017 (21). Asociada a maíz (Zea mays L.).

\section{Clave para separar las larvas de tercer estadio de Phyllophaga (sensu lato) en México Modificada de Morón et al. (2016)}

1. Raster sin septula, teges formado por un número variable de sedas hamate de longitud y grosor variables.

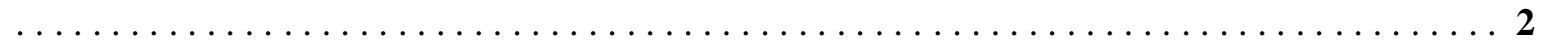

1'. Raster con septula y palidia, tegilla formada por un número variable de sedas hamate usualmente

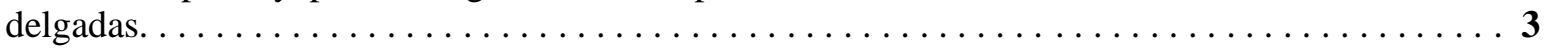

2. Proplegmatia formada por 17-19 proplegmata anchos, finamente delineados y muy próximos entre sí; teges con 6-8 sedas hamate gruesas en la parte central; anchura de la cabeza $3.4-3.7 \mathrm{~mm} . \ldots \ldots$. $\ldots \ldots \ldots \ldots \ldots \ldots \ldots \ldots \ldots \ldots \ldots \ldots \ldots \ldots \ldots \ldots \ldots \ldots \ldots \ldots$ Phyllophaga rufotestacea (Moser)

2'. Proplegmatia formada por 5-8 proplegmata anchos, muy separados entre sí; teges con 18-20 sedas hamate gruesas en la parte central; anchura de la cabeza $5.1-5.2 \mathrm{~mm} . \ldots \ldots \ldots \ldots \ldots \ldots$

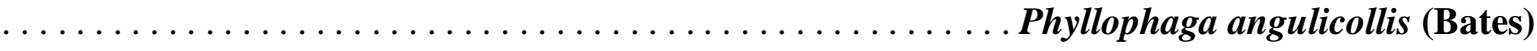

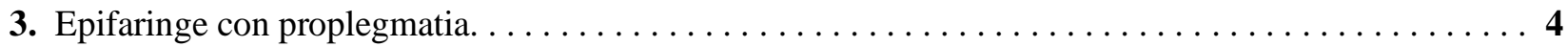

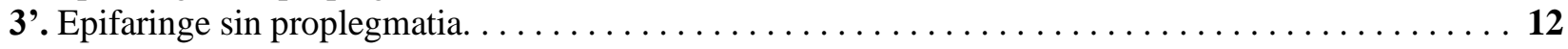

4. Placa respiratoria del segmento abdominal VII con un diámetro mayor al de la placa respiratoria del segmento VI; palidia muy recurvadas, cercanamente convergentes en ambos extremos; anchura de la cabeza $5.0-5.6 \mathrm{~mm} \ldots \ldots \ldots \ldots \ldots \ldots \ldots \ldots \ldots \ldots \ldots \ldots \ldots$ Phyllophaga ilhuicaminai Morón

4'. Placa respiratoria del segmento abdominal VII con un diámetro similar al de la placa respiratoria del

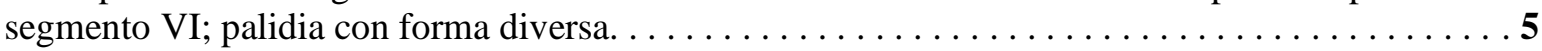

5. Palidia divergentes hacia el extremo caudal, formadas por 9-12 pali cortos y gruesos, dirigidos caudalmente, pali proximales mucho más pequeños que los distales; septula amplia, semitriangular; anchura de la cabeza $3.5-3.8 \mathrm{~mm} . \ldots \ldots \ldots \ldots \ldots \ldots \ldots$ Phyllophaga heteronycha (Bates)

5'. Palidia paralelos divergentes o convergentes con pali de tamaño similar. . . . . . . . . . . . 6

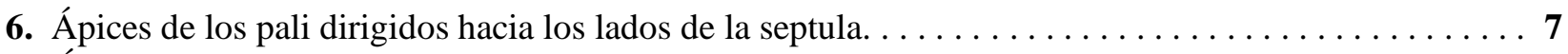

6'. Ápices de los pali dirigidos hacia la línea media de la septula . . . . . . . . . . . . . . . 8

7. Cabeza con cuatro sedas anterofrontales; cada palidium formado por 10-13 pali espiniformes, cortos y afilados; palidia ligeramente divergentes en el extremo caudal; ancho de la cabeza $4.0-4.1 \mathrm{~mm}$. . . .

Phyllophaga leonina (Bates)

7'. Cabeza con 13-14 sedas anterofrontales; cada palidium formado por 14-20 pali largos y agudos; palidia divergentes en ambos extremos; ancho de la cabeza $4.3-4.6 \mathrm{~mm}$.

Phyllophaga pubicauda (Bates)

8. Palidia poco recurvada, ligeramente convergentes en el extremo anterior, con los palis separado entre sí; cabeza con 17 sedas frontales anteriores, 5.7-5.9 mm de anchura; plegmatia formada por 10-12 y proplegmatia con 12-14 plegmata separados entre sí; cada palidium con $17-20$ pali. . . . . . . . .

Phyllophaga opaca (Moser)

8'. Cabeza parda rojiza o anaranjada, $3.7-5.1 \mathrm{~mm}$ de anchura; proplegmatia con $7-13$ plegmata muy

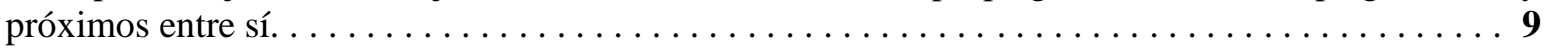


Cuate-Mozo et al.: Estados inmaduros de Phyllophaga totoreana $y \mathrm{Ph}$. opaca

9. Palidia formadas por 18-23 pali largos muy próximos entre sí, con ambos extremos ligeramente convergentes; cabeza con 6-8 sedas frontales anteriores, $4.5-4.6 \mathrm{~mm}$ de anchura. . . . . . . . . . $\ldots \ldots \ldots \ldots \ldots \ldots \ldots \ldots \ldots \ldots \ldots \ldots \ldots \ldots \ldots \ldots \ldots \ldots \ldots \ldots$ Phyllophaga multipora (Bates)

9'. Palidia casi paralelas entre sí, poco convergentes en el extremo posterior, con los pali separados entre sí.

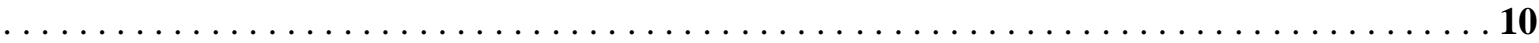

10. Cabeza con $8-9$ sedas frontales anteriores, $4.2-5.3 \mathrm{~mm}$ de anchura; plegmatia formada por $8-9$ plegmata; cada palidium con $13-16$ pali............... Phyllophaga polyphylla (Bates)

10'. Cabeza lisa, epifaringe con zygum o sin zygum. ................ 11

11. Cabeza con 7-12 sedas frontales anteriores, $4.5-5.1 \mathrm{~mm}$ de anchura; epifaringe sin zygum, plegmatia formada por 9-13 plegmata; cada palidium con 14-21 pali....... Phyllophaga ravida (Blanchard)

11'. Cabeza con 9 sedas frontales anteriores, 3.7-3.8 $\mathrm{mm}$ de anchura; epifaringe con zygum plegmatia formada por 11-13 plegmata separados entre sí; cada palidium con $12-14$ pali. . . . . . . . . . . .

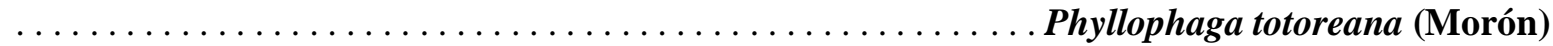

12. Palidia muy recurvadas, fuertemente convergente en sus extremos; septula ovalada. . . . . . . 13

12'. Palidia poco recurvadas o casi rectas; septula alargada, poco delimitada en los extremos. . . . . . . 14

13. Cada palidium con 9-25 pali cortos; frente poco rugosa; ancho de la cabeza $4.9-5.2 \mathrm{~mm} . \ldots \ldots \ldots$

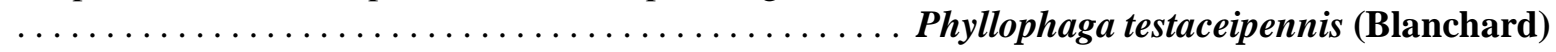

13'. Cada palidium con 18-20 pali largos; frente casi lisa; ancho de la cabeza $3.0 \mathrm{~mm}$. .

Phyllophaga macrocera (Bates)

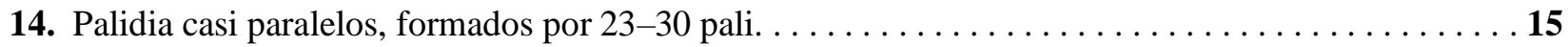

14'. Palidia ligeramente convergentes en uno o ambos extremos, formados por $11-27$ pali. . . . . . . 16

15. Septula lisa; placa respiratoria del segmento abdominal VII con un diámetro similar al de la placa del VI; maxilas con 11-18 dientecillos estriduladores; ancho de la cabeza 5.6-6.2 mm. . . . . . . . . .

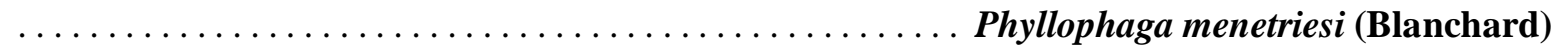

15'. Septula con estrías transversales; placa respiratoria del segmento abdominal VII con un diámetro poco mayor al de la placa del VI; maxilas con 11 dientecillos estriduladores; ancho de la cabeza $5.8 \mathrm{~mm}$. . $\ldots \ldots \ldots \ldots \ldots \ldots \ldots \ldots \ldots \ldots \ldots \ldots \ldots \ldots \ldots \ldots \ldots \ldots \ldots \ldots \ldots \ldots$ Phyllophaga vetula (Horn)

16. Palidia convergentes en ambos extremos; cabeza con dos sedas frontales posteriores.

16'. Palidia convergentes en los extremos anteriores; cabeza con cuatro sedas frontales posteriores, 4.6-4.8 mm de anchura.

Phyllophaga brevidens (Bates)

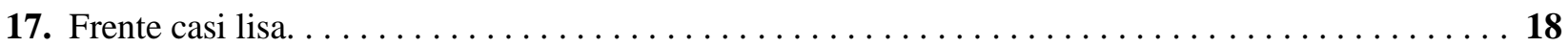

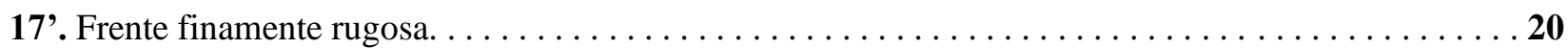

18. Acanthoparia formado por 7-10 sedas espiniformes; cada palidium con 15-27 pali cortos; ancho de la cabeza $4.1-4.8 \mathrm{~mm} . \ldots \ldots \ldots \ldots \ldots \ldots \ldots \ldots \ldots \ldots \ldots \ldots \ldots$ Phyllophaga obsoleta (Blanchard)

18'. Acanthoparia formado por $9-12$ sedas espiniformes. . . . . . . . . . 19

19. Cabeza con 13-15 sedas frontales posteriores, $4.5-4.8 \mathrm{~mm}$ de anchura; palidia con $13-21$ pali. .....

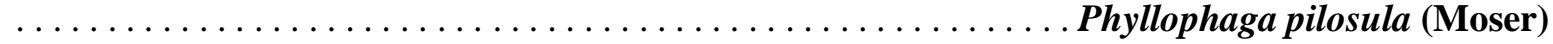

19'. Cabeza con 12 sedas frontales posteriores, $4.7 \mathrm{~mm}$ de anchura; palidia con 19-21 pali. . .......... 
20. Cabeza con 10-12 sedas frontales anteriores, 4.9-5.6 mm de anchura; plegmata formada por 9-10 plegmatia; palidium con $24-26$ pali. ..................... Phyllophaga misteca (Bates)

20'. Cabeza con 8-14 sedas frontales anteriores, $6.1-6.7 \mathrm{~mm}$ de anchura; plegmata formada por 10-15 plegmatia; palidium con $11-18$ pali. .................. Phyllophaga tenuipilis (Bates)

\section{LITERATURA CITADA}

Aragón, A., Morón, M. A. (2004) Un método de cría para gallinas ciegas rizófagas del género Phyllophaga (Coleoptera: Melolonthidae). Pp. 109-118. En: N. Bautista-Martínez, H. Bravo M., C. Chavarin P. (Eds.). Cría de insectos plaga y organismos benéficos. Colegio de Posgraduados. CONABIO. Montecillo, Texcoco, Estado de México.

Böving, A. (1936) Description of the larvae of Plectis aliena Chapin and explanation of new terms applied to the epipharynx and raster. Proceedings of the Entomological Society of Washington, 38, 169185.

Lacroix, M. (2014) Melolonthidae Malgaches (Coleoptera, Scarabaeoidea). Editions Marc Lacroix, Collection Hannetons, Paris, 260 pp.

Lavelle, P., Maury, M. E., Serrano, V. (1981) Estudio comparativo de la fauna del suelo en la región de Laguna Verde, Veracruz. Época de lluvias. Pp. 75-105. En: P. Reyes-Castillo (Ed.). Estudios ecológicos en el trópico mexicano. Instituto de Ecología A.C. México, D.F.

Lugo-García, G. A., Morón, M. A., Reyes-Olivas, A., Aragón-García, A., Ortega-Arenas, L. D., CotaSánchez, H. (2011) Riqueza de escarabajos con hábitos nocturnos (Coleoptera: Scarabaeoidea) en islas de las bahías de Ohuira y Nacachiste, Sinaloa, México. Dugesiana, 18 (1), 1-10.

Lugo-García, G. A., Morón, M. A., Aragón, G. A., Ortega, A. L. D., Reyes-Olivas, A., Sánchez, B. H. (2013) Especies nocturnas de Scarabaeoidea (Coleoptera: Polyphaga) en el norte de Sinaloa, México. Revista Colombiana de Entomología, 39 (1), 9-104.

Lugo-García, G. A., Morón, M. A., Aragón-Sánchez, M., Reyes-Olivas, Á., Sánchez-Soto, B. H., Sauceda-Acosta, C. P. (2017) White grubs species (Coleoptera: Melolonthidae) associated with sesame (Sesamun indicum L.) crops in Sinaloa, Mexico. Agrociencia, 51 (7), 799-811.

Morón, M. A. (2003) Diversidad, distribución e importancia de las especies de Phyllophaga Harris en México (Coleoptera: Melolonthidae). Pp. 1-27. En: A. Aragón G., M. A. Morón, A. Marín J. (Eds.). Estudios sobre Coleópteros del suelo en América. Publicación especial de la Benemérita Universidad Autónoma de Puebla. Puebla, México.

Morón, M. A. (2006) Revisión de las especies de Phyllophaga (Phytalus) grupos obsoleta y pallida (Coleoptera: Melolonthidae: Melolonthinae). Folia Entomológica Mexicana, 45 (Supl. 1), 1-104.

Morón, M. A., Rojas-Gómez, C. V., Arce, R. (2016) Los estados inmaduros de Phyllophaga heteronycha, $P$. leonina y $P$. angulicollis (Coleoptera: Melolonthidae: Melolonthinae). Revista Mexicana de Biodiversidad, 87, 933-943.

http://dx.doi.org/10.1016/j.rmb.2016.07.014

Ramírez-Salinas, C., Castro-Ramírez, A. E. (2015) Descripción del tercer estadio larval de tres especies de Ceraspis pilatei (Harold, 1863), Diplotaxis puberea (Bates, 1887) y Phyllophaga (s.str.) multipora (Bates, 1888) (Coleoptera: Melolonthidae) de Chiapas, México. Dugesiana, 22, 251-259.

Rivera-Gasperín, S. L., Morón, M. A. (2013) Análisis filogenético del subgénero Phyllophaga (Triodonyx) (Coleoptera: Melolonthidae: Melolonthinae). Revista Mexicana de Biodiversidad, 84 (3), 802-817.

Rivera-Gasperín, S. L., Morón, M. A. (2017a) Phylogenetic relationships within Phyllophaga Harris (sensu lato) (Coleoptera: Melolonthidae, Melolonthinae) with emphasis on Listrochelus Blanchard. Neotropical Entomology, 46, 524-536. http://dx.doi.org/10.1007/s13744-017-0482-6 
Cuate-Mozo et al.: Estados inmaduros de Phyllophaga totoreana y Ph. opaca

Rivera-Gasperín, S. L., Morón, M. A. (2017b) Relaciones filogenéticas de las especies de Chlaenobia con otros miembros de Phyllophaga (s. lato) (Coleoptera: Melolonthidae: Melolonthinae). Revista Mexicana de Biodiversidad, 88, 592-607. 